\title{
Modeling, Analysis, and Comparison of Nanotechnology System: A Graph-Theoretic Approach
}

\section{Tanvir Singh ( $\sim$ tanvirsingh3@gmail.com )}

DR. B.R. Ambedkar National Institute of Technology Jalandhar, India https://orcid.org/0000-00031952-6275

\section{Research Article}

Keywords: Nanotechnology system, Digraph approach, Structural modeling, Permanent function, Integrative Analysis

Posted Date: January 5th, 2021

DOl: https://doi.org/10.21203/rs.3.rs-136389/v1

License: (c) (i) This work is licensed under a Creative Commons Attribution 4.0 International License. Read Full License 


\title{
Modeling, Analysis, and Comparison of Nanotechnology System: A Graph-Theoretic
}

\section{Approach}

\section{Tanvir Singh ${ }^{*}$}

1Department of Mechanical Engineering, DR. B.R. Ambedkar National Institute of Technology, G.T Road Bypass Amritsar, Jalandhar, Punjab-144011, India

*Author to whom correspondence should be addressed E-mail: tanvirsingh3@gmail.com, Tel: +918146426766 (Tanvir Singh)

\begin{abstract}
Nanotechnology can create many new nanomaterials and nanodevices with a vast range of applications, such as in medicine, electronics, biomaterials, and energy production, etc. An attempt is made to develop an integrated systems model for the structure of the nanotechnology system in terms of its constituents and interactions between the constituents and processes, etc. using graph theory and matrix algebra. The nanotechnology system is first modeled with the help of graph theory, secondly by variable adjacency matrix and thirdly by multinomial (which is known as a permanent function). The permanent function provides an opportunity to carry out a structural analysis of nanotechnology system in terms of its strength, weakness, improvement, and optimization, by correlating the different systems with its structure. The physical meaning has been associated with each term of the permanent function. Different structural attributes of the nanotechnology system are identified concurrently to reduce cost, time for design and development, and also to develop a graph-theoretic model, matrix model, and multinomial permanent model of nanotechnology system. The top-down approach for a complete analysis of any nanotechnology systems is given. The general methodology is presented for the characterization and comparison of two nanotechnology systems.
\end{abstract}

Keywords: Nanotechnology system, Digraph approach, Structural modeling, Permanent function, Integrative Analysis 


\section{Introduction}

Recent advances in the development of advanced manufacturing techniques have the result that the machined and manufactured components are no longer restricted to micrometer scale but now fabricated at the nanometre scale. For the past few decades, nanotechnology has greatly influenced all science and engineering branches including physics, electronics [1], civil engineering [2], material engineering [3], etc. Much of the fascination with nanotechnology stems from these quantum and surface phenomena that matter exhibits at the nanoscale [4]. Nanotechnology is considered a key technology for the future. Nanotechnology entails the applications of fields of science as diverse as surface science, micro-fabrication, etc. Nevertheless, progress on innovative materials and methodologies has been demonstrated with some patents granted about new nanomanufacturing devices for future commercial applications, which progressively helps in the development of nanorobots with the use of embedded nanobioelectronics concepts $[5,6]$. On the other hand, nanotechnology raises many of the same issues as any new technology, including concerns about the toxicity and environmental impact of nanomaterials. The complexity both in terms of geometry and chemical composition of nanomaterial systems requires the use of a range of different approaches from semi-empirical calculations to handle very complex systems through to "abinitio" approaches for more precise results on easier to handle systems. Micro- and nanofabrication techniques have revolutionized the pharmaceutical and medical fields as it offers the possibility for highly reproducible mass-fabrication of systems with complex geometries and functionalities, including novel drug delivery systems and biosensors. Scientists debate the future implications of nanotechnology. Now a day's researchers have narrowed their research focus down to the nanometre dimension that is referred to as nano-level. Nanomanipulation of nanoparticles has been a widespread interest for the last years, and dynamic modeling is a basic tool for understanding the pushing procedure in real-time. Much work has been done during the last two decades in modeling and analyzing the nanotechnology system for various characteristics. System engineering has evolved as a novel approach to model the nanotechnology system. It is proposed that the structural/system modeling technique acts as a framework through which components, attributes, inter-relationship, and inter-dependencies concurrently within and across the system are expressed. Our contribution is to propose a mathematical model, which analyzes without loss of generality all the flow, information, control, semantics, static and dynamic behaviors of systems and sub-systems using graph theory, matrix algebra, and permanent function. The present work deals with the modeling and analysis of nanotechnology system. The methodology is so strong that it analyzes all aspects of nanotechnology concurrently without losing any information and optimizes the characteristics associated with it. Several research groups have reported precise manipulation 
of the nanoparticle to form two or even three-dimensional pattern with an appropriate chemical or thermal process. Zesch, W.et al. [7] have developed the piezoelectric model and inertial drive model for multi-degree-of-freedom micro-positioning using stepping principles. Darby, AP., and Pellegrino, S. [8] have realized both linear and piezoelectric stick-slip actuators to prove the stick-slip mechanism as a valid actuation principle. Shanian and Savadogo [9] have applied the TOPSIS method as multiple-criteria decision support analysis for material selection of metallic bipolar plates for polymer electrolyte fuel cells. Schaffer et al. [10] have introduced the electrically induced nanopatterning techniques utilize electrostatic interactions between a thin dielectric material liquid film and an electric field gradient to produce lateral patterns and structures at the nanometre scale. Rao et al. [11] presented an improved compromise ranking method for material selection. Bhaduri, D et al. [12] have developed a tool in MATLAB for modeling of logic gates at the nanolevel. The model handles discrete energy distributions at the inputs and interconnects of any specified architectural configuration. Offodile et al. [13] developed a coding and classification system which was used to store robot characteristics in a database and then selected a robot using economic modeling. Buehler, M.J. et al. [14] have developed mesoscopic models in which the coarse-grained particles allow one to explicitly reproduce the nanostructure of the material. Barnard, A.S. et al. [15] have modeled nanoparticles approaches to morphology and evolution, but, many questions regarding their physical, chemical, electric, magnetic, and optical properties remain unanswered. For example, with regards to the spray deposition method, it is not fully known to what extent and in what ways, nanoparticles, interact with each other after making contact with the substrate. This is one of the reasons why simulation studies are very helpful. Carpenter, D. T et al. [16] have found out that the hundreds of particles are measured to present statistically reliable mean size data. For instance, it has been proposed to measure 500-1000 grains for an optimum sample size Amrute et al. [17] have identified firstly the useful application of nanomachines might be in medical technology and which could be used to identify and destroy cancer cells by Patel et al. [18], Wang et al. [19]. Fan et al. [20] have proposed rapid prototyping combines various nanofabrication techniques for the generation of complex geometrical patterns, multi-layered structures, with chemical functionality. The authors are not aware of any study that integrates all the subsystems and systems of nanotechnology. Researchers have identified that the performance of any nanotechnology system is a function of its basic structure (i.e. fabrication, manipulation, attributes, reliability, availability, etc.). The understanding of systems structure and its connectivity and interactions between different systems and down to component level is useful for estimating the contribution of different attributes of the performance of the system. Currently, no effective mathematical model is present for studying these aspects concerning each other or independently. An attempt is made in the paper to represent the nanotechnology 
system mathematically and a methodology to model the complete structure of the nanotechnology system. It is achieved with the help of graph theory, matrix algebra, and permanent function. Concurrent engineering considers all the aspects of the system concurrently to reduce cost, time for design, and development. The proposed method is also doing the same thing in the area of nanotechnology. The tool has so far been used by various authors to study a subsystem for a particular attribute of the performance of a system in nuclear plant Sacks et al. [21], selection of rolling elements of bearings Seghal et al. [22]. Venkataswamy et al. [23] have proposed a graph theoretic approach for system and structural analysis of an automobile vehicle. Mohan et al. [24] have proposed systems modeling of a coal-based steam power plant. Gandhi et al. [25] have applied digraph and matrix approach in Failure mode effect analysis (FEMA), Sandeep et al. [26] have applied digraph approach to total quality management (TQM) evaluation of an industry, but so far graph theory approach has not been used to model and analyze nanotechnology system with special emphasis to their constituents and their interactions. Our mathematical model preserves all inter-relationships, interdependencies, interactions within and across the systems, and sub-systems in a single multinomial function. The model permits us to evaluate various characteristics such asTopology, physical attributes, Nanometric system, manipulation, etc., associated with the nanotechnology system. In the previous works, researchers were mainly concern about the evaluation of systems/subsystems (components) and interactions independently. The limitation of these approaches results in not fully optimizing the overall system characteristics as the approaches do not analyze or evaluate all information (components and interactions) together. Our contribution is the major break-through in optimizing the overall system characteristics by giving special emphasis to evaluate and analyze structural modeling aspects of the nanotechnology system based on the system engineering approach concurrently.

\section{Identification of subsystems of the nanotechnology system}

For the description of the methodology, five subsystems have been identified namely, nanomaterial, nanofabrication, nanostructure, nanomachining, and nanomanipulation subsystems. All important criterions governing the nanotechnology behavior reflected in these subsystems. These subsystems are not universal and there are some variations for modeling specific nanotechnology systems. However, the proposed model can consider any such variations and is suitable for modeling any particular nanotechnology system structure. The following sections discuss the importance of each of these identified subsystems of the nanotechnology system.

> Nanomaterial Subsystem:-Nanomaterial subsystem is that field that takes into account, the material science-based approach to nanotechnology. The important aspect of 
nanotechnology is the vastly increased ratio of surface area to the volume present in many nanoscale materials, which makes possible new quantum mechanical effects. Nanomaterial with morphological features and have special properties stemming from their nanoscale dimensions. Various types of nanomaterial subsystems are to be considered for modeling general nanotechnology systems are given as attributes, properties, reactivity, nucleations, characteristics, compositions, agglomeration, structures, etc. All the subsystems of nanomaterials are integrated in such a way, that every aspect of each subsystem has put their importance to improve the characteristic features of new nanomaterials.

$>$ Nanofabrication Subsystem: Nanofabrication subsystem is mainly concerned with the study and applications of fabricating nanometre-scale structures meaning patterns. Various lithographic techniques are used to fabricate nanodevices such as nanolithography, which is used for the fabrication of leading-edge semiconductor integrated circuits (nano-circuitry) or nano-electromechanical systems (NEMS). It is a promising tool for patterning at the deep nanometre-scale. The atomic force microscopic nanolithography (AFMN) is a chemomechanical surface patterning technique that uses atomic force microscope Davis et al. [27]. Nanofabrication subsystem deals with the techniques that are used to produce and fabricate nanoscale devices.

$>$ Nanostructure Subsystem:-Nanostructure subsystem is mainly concerned with the versatile techniques that are used to visualize relatively large samples and various dimensional measurements are to be taken and various comparison analyses are performed. For topology and geometries of nanostructures, the imaging modes allow the mapping of surface properties. These include atomic force microscopy (AFM), pulsed force microscopy (PFM), scanning tunneling microscopy (STM), etc. which maps the mechanical properties. Measurement and integration techniques play a very important role in measuring dimensions and to integrate them. It focuses on the unique properties of nanoscale structures and materials that do not exist in structures of the same material composition but at other scale ranges.

> Nanomachining Subsystem:-It is mainly dealt with a better understanding of nanometric physics and the development of its controllable techniques to meet the advanced requirements of nanotechnology and nanoscience. Nanometric machining is indispensable to manufacture complex micro and miniature structures, components, and products in a variety of engineering materials and it is applied in bulk machining of silicon, aluminum substrates for computer memory, etc. It is very promising in the production of sensors, actuators, etc. The indispensable advantage of nanometric machining is its applicability to manufacturing 3D complex components/devices. Therefore, it is undoubtedly one of the major enabling technologies for the commercialization of nanotechnology in the future. 
> Nanomanipulation Subsystem:-Nanomanipulation is a technology to manipulate small object sized in nanometre to submicron scale. Optical tweezers are one of the nanomanipulation techniques that investigates pico-newton to Femto-newton force exerted on microscopic objects. Nanomanipulation of nanoparticles has been widespread of interest for the last years and dynamic modeling is a basic tool for understanding the pushing procedure in real-time. Nanomanipulators have been built to perform nanomanipulation studies under vacuum inside a scanning electron microscope (SEM). Researchers at Harvard University have been created nanoscale grasping device ideal for measuring and manipulating molecular structures. So the nanomanipulation system helps to integrate the nanotechnology system and for the development of nanoproducts for various applications.

\subsection{Graph-theoretic representation}

Nanotechnology is considered to be a collection of several basic constituents, e.g. Nanomaterial, Nanofabrication, Nanostructure, Nanomachining, and Nanomanipulation is shown in Figure 1. These constituents are connected through different forms of bonding and interactions. The constituents and interactions forming nanotechnology system are shown in Figure 2 with the help of a schematic diagram where blocks show constituents, lines show connectivity/interactions and arrows show directional bonding/interactions. However, a schematic diagram is a good representation of the nanotechnology system for better understanding its structure, but it is not a mathematical entity. However, it is not possible to derive different results as no mathematical operations are carried out. It is proposed to introduce a mathematical representation of nanotechnology system (i.e. constituents cum process design and manufacturing) attributes in an attempt to develop systems model for analysis and synthesis of the nanotechnology system which is useful to carry out analysis of existing product concurrently for improvement and cost reduction apart from other benefits in the competitive world Deo et al. [28] and Robinson and Foulds [29]. Nanotechnology is considered to be system $[\mathbf{N}, \mathbf{I}]$ of its constituents set $[\mathbf{N}]=\left[\mathbf{N}_{1}, \mathbf{N}_{2}, \ldots . . . . \mathbf{N}_{\mathbf{i}}\right]$ and interactions/connectivity set $[\mathbf{I}]=\left[\mathbf{I}_{\mathbf{1}}, \mathbf{I}_{2}, \ldots \ldots . . . \mathbf{I}_{\mathbf{j}}\right]$, where $\mathbf{N}_{\mathbf{i}}$ represents $\mathbf{i}^{\text {th }}$ constituent (and attributes associated with it), while $\mathbf{I j}$ represents $\mathbf{j}^{\text {th }}$ interactions/connectivity between two corresponding constituents of the nanotechnology system. For the representation of the structure of the nanotechnology system, it is quite logical to select graph theory Deo et al. [28] and for mathematical representation of the structure of the nanotechnology system, it is logical to select matrix algebra Jurkat and Ryser [30]. Graph $\mathbf{G}$ is a set, if vertex and edge [V, E], where vertex set $[\mathbf{V}]=\left[\mathbf{V}_{\mathbf{1}}, \mathbf{V}_{2} \ldots \ldots . . . . . \mathbf{V}_{\mathbf{n}}\right]$ and edge set $[\mathbf{E}]=\left[\mathbf{e}_{1}, \mathbf{e}_{2} \ldots \ldots \ldots . . \mathbf{e}_{\mathbf{m}}\right]$ are its subset. To represent the nanotechnology system mathematically, let the constituents of nanotechnology are represented by vertex set [V] of the graph, and connectivity/interactions between different 
constituents are represented by edge set [E]. Any directional property existing in connectivity/interactions is represented by a directed edge instead of an undirected edge. To develop an algorithm, let us assume that there are five constituents $\left[\mathbf{N}_{\mathbf{1}}, \mathbf{N}_{\mathbf{2}}, \mathbf{N}_{3}, \mathbf{N}_{4}, \mathbf{N}_{\mathbf{5}}\right.$ ] forming nanotechnology system as shown in Figure 3 are represented by five vertices $\left[\mathbf{v}_{\mathbf{1}}, \mathbf{v}_{\mathbf{2}}, \mathbf{v}_{\mathbf{3}}, \mathbf{v}_{\mathbf{4}}, \mathbf{v}_{\mathbf{5}}\right]$, i.e. constituent $\mathbf{N}_{\mathbf{i}}$ is represented by vertex $\mathbf{V}_{\mathbf{i}}$. Let any connectivity/interactions existing between the constituents $\mathbf{N}_{\mathbf{i}}$ and $\mathbf{N}_{\mathbf{j}}$, i.e. between $\mathbf{v}_{\mathbf{i}}$ and $\mathbf{v}_{\mathbf{j}}$ are represented by edge $\mathbf{e}_{\mathbf{i j}}$ (an edge existing between $\mathbf{v}_{\mathbf{i}}$ and $\mathbf{v}_{\mathbf{j}}$ ). If it is assumed that all the five constituents are interacting with each other and have general directional characteristics, then the nanotechnology system is having graphtheoretic representation with $\mathbf{e}_{\mathbf{i j}} \neq \mathbf{e}_{\mathbf{j i} .}$. The $\mathbf{e}_{\mathbf{i j}} \neq \mathbf{e}_{\mathbf{j i}}$ means that the influence of $\mathbf{i}^{\text {th }}$ vertex on the $\mathbf{j}^{\text {th }}$ vertex is not equal to the influence of $\mathbf{j}^{\text {th }}$ vertex on $\mathbf{i}^{\text {th }}$ vertex. If the directional property is not significant, the nanotechnology system is represented by an undirected graph, in that case, $\mathbf{e}_{\mathrm{ij}}=$ $\mathbf{e}_{\mathbf{j i}}$. In many nanotechnology systems like the nanofabrication system, some constituents are not connected or interacting with each other. In that case, no edges exist between those vertices. In some of the nanotechnology systems, like nanomanipulation system there are no directional interactions present between two constituents i.e. vertices these edges are undirected, while other edges are directed. It means a graph-theoretic representation of the nanotechnology system consists of both the directed and undirected edges. The nanotechnology system essentially consists of five subsystems. The schematic diagram shown in Figure 2 is a nonmathematical entity and it is now represented by a mathematical entity called nanotechnology system digraph which is shown in Figure 3. If the directional interactions, i.e. $\mathbf{P}_{\mathbf{i j}}=\mathbf{P}_{\mathbf{j i}}$ are not considered, then undirected graph representation of the nanotechnology system is done. One way or two-way interactions and degree of influence are not considered in the undirected graph, because of $\mathbf{P}_{\mathrm{ij}}=\mathbf{P}_{\mathrm{ji}}$.

\subsection{Matrix representation}

Since digraph is visual representation helps in visual analysis to a limited extent only. To establish a computer-friendly representation for the nanotechnology system, a digraph is represented in matrix form. The undirected graph representation of the nanotechnology system is shown in Figure 4. The matrix representation permits to carry out storage, retrieval, and analysis of the nanotechnology system. Let, it represents digraph of ' $\mathbf{n}$ ' sub-systems leading to

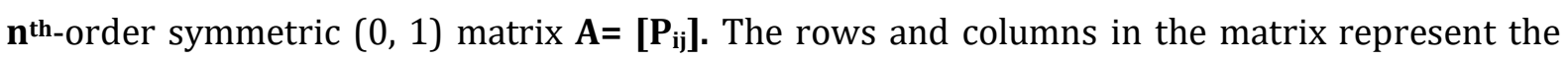
vertices/subsystem attributes, i.e. $\mathbf{P}_{\mathbf{i j}}$ represents the interactions of the $\mathbf{i}^{\text {th }}$ subsystem with the $\mathbf{j}^{\text {th }}$ subset.

$$
P_{i j}=\left\{\begin{array}{c}
1, \text { if subsystem }, i \text { is connected to subsystem } j \\
0, \text { otherwise }
\end{array}\right.
$$


Generally, $\mathbf{P}_{\mathbf{i j}} \neq \mathbf{P}_{\mathbf{j i}}$ as nanotechnology attributes are directional and $\mathbf{P}_{\mathrm{ii}}=\mathbf{0}$, when the subsystem is not interacting with itself (sometimes if it exists, for the moment let us assume it does not exist). The nanotechnology system matrix is square and nonsymmetric and is analogous to the adjacency matrix in graph theory. The nanotechnology system matrix (NTSM) representing the digraph is written as:-

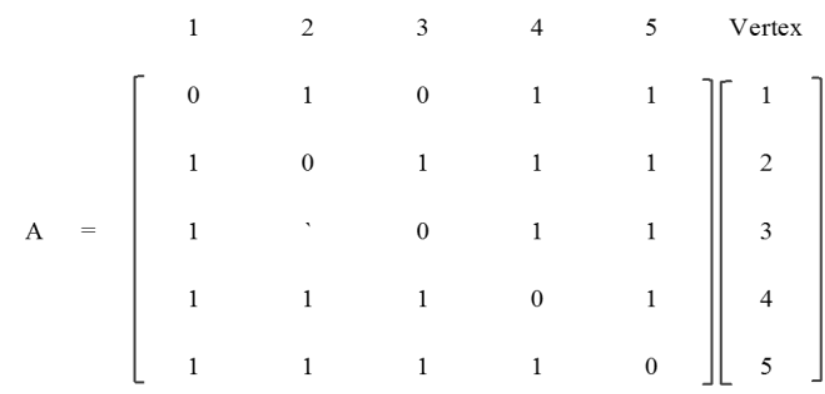

In the above matrix $A$, the values of $\mathbf{P}_{\mathbf{I I}}, \mathbf{P}_{\mathbf{1 3}}, \mathbf{P}_{\mathbf{3 2}}$ are zero by assumption. Off diagonal elements with value 0 and 1 represents the interdependency and connectivity between subsystems $\left(\mathbf{P}_{\mathbf{i}}\right.$, i=1, 2, 3....., 5) of nanotechnology system represented by Equation (1). The diagonal elements are 0 because there is no interdependency/connectivity of any subsystem with itself. To characterize the nanotechnology system, the characteristic matrix (NTSCM) is defined.

\subsubsection{Nanotechnology System- Characteristic Matrix (CM-NANOTECHNOLOGY)}

Let us consider, an identity matrix I and $\mathrm{P}$ as a variable representing the nanotechnology system. The characteristics matrix already used in mathematics [30] to characterize different systems. The nanotechnology system characteristics matrix, B for digraph is expressed as [PI A], where A is the Nanotechnology system matrix (NSM) represented by Equation (2).

$$
B=\left[\begin{array}{cccccc}
1 & 2 & 3 & 4 & 5 & \text { Vertex } \\
P & -1 & 0 & -1 & -1 \\
-1 & P & -1 & -1 & -1 \\
-1 & 0 & P & -1 & -1 \\
-1 & -1 & -1 & P & -1 \\
-1 & -1 & -1 & -1 & P
\end{array}\right]\left[\begin{array}{c}
1 \\
2 \\
3 \\
4 \\
5
\end{array}\right]
$$

In the above matrix B, values of all the diagonal elements are the same (i.e. all nanotechnology subsystems are assumed to be identical, which would or would not be true in practice since all nanotechnology subsystems have different characteristics depending on various parameters affecting them). Interdependencies between the subsystems have been assigned values of 0 and 1 depending on whether it is there or not. But it does not represent the varying degree of 
influence of one subsystem over the other subsystems. To consider that, another matrix called nanotechnology system variable characteristics matrix (NTSVCM) is proposed.

\subsubsection{Nanotechnology System Variable Characteristics Matrix (VCM-NANOTECHNOLOGY)}

The nanotechnology system variable characteristics matrix takes into consideration the effect of different nanotechnology subsystems and their varying degrees of interactions. The digraph is considered for defining VCM-NANOTECHNOLOGY. Let, $\mathbf{P}_{\mathbf{i}} \mathbf{S}$ and $\mathbf{P}_{\mathrm{ij}} \mathbf{S}$ represent nodes and edges respectively in the digraph. Consider, square matrix $\mathrm{C}$ with off-diagonal elements $\mathrm{P}_{\mathrm{ij}}$ representing varying interactions between the nanotechnology subsystems, i.e., instead of 1 (as in the matrix of Equation (1)).

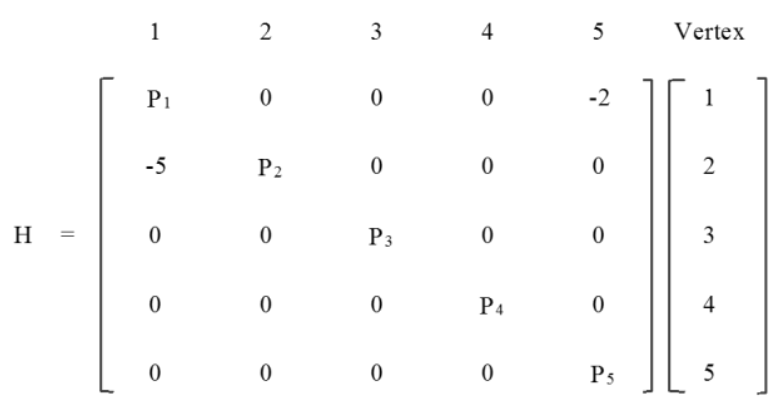

Another matrix, $\mathrm{D}$ is taken with diagonal elements $\mathbf{P}_{\mathbf{i}}, \mathbf{i}=\mathbf{1}, \mathbf{2}, \ldots \ldots . \mathbf{5}$, where, $\mathrm{P}_{\mathrm{i}}$ represents five different subsystems. All are distinct systems $P_{i}$ represents the varying inheritance of structural attributes in these subsystems. Considering matrices C and D, VCM-NANOTECHNOLOGY is expressed as $\mathbf{H}=$ [D - C]. The matrix, $\mathrm{H}$ permits us to represents complete information about all the five subsystems and interactions amongst them. The information is useful for the analysis, design, and development of new nanoproducts at the conceptual stage or optimization purposes. The matrix provides a powerful tool through its determinant called variable characteristics nanotechnology multinomial (VC-NANOTECHNOLOGY MULTINOMIAL). The characteristics of the system represent the complete nanotechnology system, considering the effect of nanotechnology subsystems and their interactions. Due to the consideration of selective interactions between the nanotechnology subsystems some of the diagonal elements in matrix H, I .e., in Equation (3) are zero.

The determinant of the matrix, $\mathrm{H}$, carries positive and negative signs with some of its terms. The multinomial in symbolic terms contains complete information on the nanotechnology system. If the symbols $\mathbf{P}_{\mathbf{i}}$ and $\mathbf{P}_{\mathbf{i j}}$ with their numerical values are replaced then the information is lost. Hence, complete information on the nanotechnology system is not obtained as some of them are lost due to the addition and subtraction of numerical values of diagonals and offdiagonal elements (i.e. $\mathbf{P}_{\mathbf{i}} \mathbf{S}$ and $\mathbf{P}_{\mathrm{ij}} \mathbf{s}$ ). The multinomial's of the matrix $\mathrm{H}$ in Equation (3) does not provide complete information concerning nanotechnology system under certain conditions. To 
avoid the loss of structural information during mathematical processing, another term nanotechnology system variable permanent matrix (VPM-NANOTECHNOLOGY) is introduced.

\subsubsection{Nanotechnology System Variable Permanent Matrix (VPM-NANOTECHNOLOGY)}

To develop a unique and comprehensive model of nanotechnology system represented by a schematic diagram and digraph another entity called a permanent matrix frequently used in combinational mathematics is proposed. Let, the permanent matrix of five-subsystems of nanotechnology system is defined by Equation (4) as:-

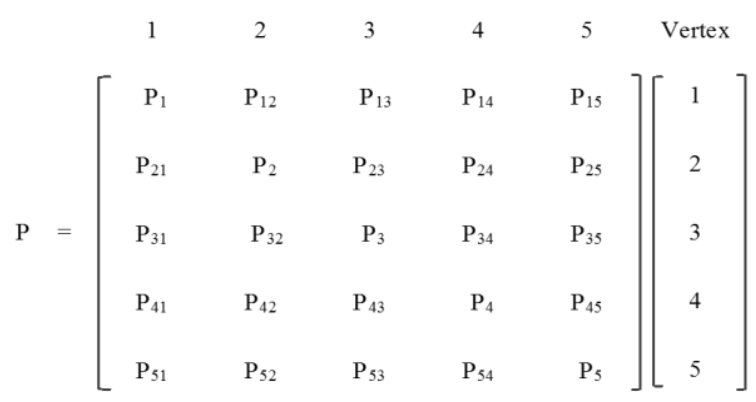

The variable permanent nanotechnology matrix (VPM-NANOTECHNOLOGY) corresponding to five subsystems shown in a digraph is given by Equation (5) as:

$$
\mathrm{E}=\left[\begin{array}{cccccc}
1 & 2 & 3 & 4 & 5 & \text { Vertex } \\
\mathrm{P}_{1} & \mathrm{P}_{12} & 0 & \mathrm{P}_{14} & \mathrm{P}_{15} \\
\mathrm{P}_{21} & \mathrm{P}_{2} & \mathrm{P}_{23} & \mathrm{P}_{24} & \mathrm{P}_{25} \\
\mathrm{P}_{31} & 0 & \mathrm{P}_{3} & \mathrm{P}_{34} & \mathrm{P}_{35} \\
\mathrm{P}_{41} & \mathrm{P}_{42} & \mathrm{P}_{43} & \mathrm{P}_{4} & \mathrm{P}_{45} \\
\mathrm{P}_{51} & \mathrm{P}_{52} & \mathrm{P}_{53} & \mathrm{P}_{54} & \mathrm{P}_{5}
\end{array}\right]\left[\begin{array}{c}
1 \\
2 \\
3 \\
4 \\
5
\end{array}\right]
$$

The diagonal elements, $\mathbf{P}_{1}, \ldots . . \mathbf{P}_{5}$ represents the contributions of five critical subsystems in producing nanotechnology system and off-diagonal elements represent interdependences of subsystems quantitatively in the matrix without any loss of information in multinomial representation.

\subsection{Permanent function representation}

Both digraph and matrix representation is not as unique as these models change by changing the labeling of nodes. To develop a unique representation of the nanotechnology system, independent of labeling the permanent function of the matrix, the VPM-NANOTECHNOLOGY is proposed for this purpose. The permanent function is similarly obtained from the matrix to its determinant. The negative sign appears in the expansion of determinant in variable permanent function and positive signs replace these negative signs. The computation process results in 
multinomial (Equation (6a) that's every term has its physical significance related to the nanotechnology system. The multinomial representation includes all the information regarding various constituents as subsystems, sub-sub systems, and interactions amongst them. The variable permanent function for nanotechnology system modeled as five subsystem nanotechnology system is derived from matrix representation as shown in Equation (4). The variable permanent function for a nanotechnology system is given as:

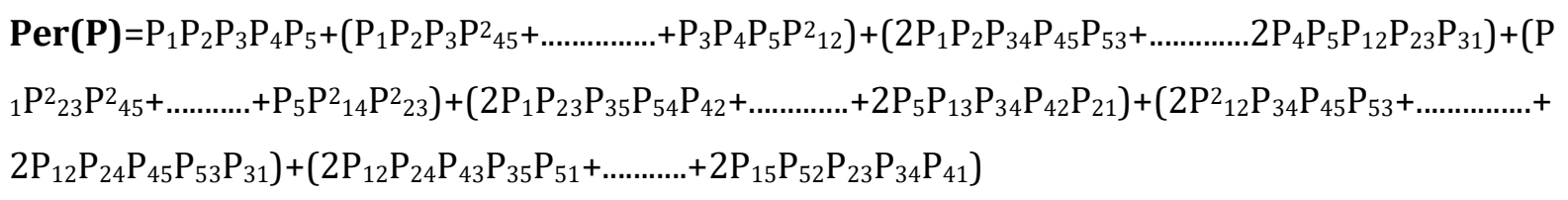

The Equation (6a) is for the general nanotechnology system modeled for five subsystems, which contains 120 terms. But the permanent function for matrix Equation (5) corresponds to Figure 3 reduces to 78 terms because the values of variables $\mathbf{P}_{\mathbf{1 3}}$ and $\mathbf{P}_{\mathbf{3 2}}$ are 0 , which means interactions are absent. The variable permanent function for nanotechnology system is given by:

$$
\begin{aligned}
& \operatorname{per}(\mathrm{E})=\mathrm{P}_{1} \mathrm{P}_{2} \mathrm{P}_{3} \mathrm{P}_{4} \mathrm{P}_{5}+\left(\mathrm{P}_{1} \mathrm{P}_{2} \mathrm{P}_{3} \mathrm{P}_{45} \mathrm{P}_{54}+\ldots \ldots \ldots+\mathrm{P}_{2} \mathrm{P}_{3} \mathrm{P}_{4} \mathrm{P}_{15} \mathrm{P}_{51}\right)+\left(\mathrm{P}_{1} \mathrm{P}_{2} \mathrm{P}_{34} \mathrm{P}_{45} \mathrm{P}_{53}+\ldots \ldots . .+\mathrm{P}_{2} \mathrm{P}_{4} \mathrm{P}_{15} \mathrm{P}_{53} \mathrm{P}_{31}\right)+\left(\mathrm{P}_{1}\right. \\
& \left.\mathrm{P}_{24} \mathrm{P}_{42} \mathrm{P}_{35} \mathrm{P}_{53}+\ldots \ldots \ldots+\mathrm{P}_{5} \mathrm{P}_{12} \mathrm{P}_{21} \mathrm{P}_{34} \mathrm{P}_{43}\right)+\left(\mathrm{P}_{1} \mathrm{P}_{24} \mathrm{P}_{43} \mathrm{P}_{35} \mathrm{P}_{52}+\ldots \ldots \ldots+\mathrm{P}_{5} \mathrm{P}_{14} \mathrm{P}_{42} \mathrm{P}_{23} \mathrm{P}_{31}\right)+\left(\mathrm{P}_{12} \mathrm{P}_{21} \mathrm{P}_{34} \mathrm{P}_{45} \mathrm{P}_{53}+\ldots \ldots .+\right. \\
& \left.\mathrm{P}_{24} \mathrm{P}_{42} \mathrm{P}_{15} \mathrm{P}_{53} \mathrm{P}_{31}\right)+\left(\mathrm{P}_{12} \mathrm{P}_{24} \mathrm{P}_{43} \mathrm{P}_{35} \mathrm{P}_{51}+\ldots \ldots \ldots . .+\mathrm{P}_{15} \mathrm{P}_{52} \mathrm{P}_{24} \mathrm{P}_{43} \mathrm{P}_{31}\right) .
\end{aligned}
$$

The Equation (6b) uniquely represents the nanotechnology system. Every term of these Equations represents the subset of the nanotechnology system. It is possible to write these Equations simply by visual inspection of the nanotechnology system. To achieve the objective, the permanent function of Equation $(6 \mathrm{a} \& 6 \mathrm{~b})$ is written in standard form as $(\mathbf{N}+\mathbf{1})$ groups. All these distinct combinations of subsystems and interactions of the nanotechnology system are shown graphically in Figure 5. The multinomial i.e. the permanent function, when written in $(\mathbf{N + 1})$ groups present an exhaustive way for analysis of nanotechnology at different levels. It helps in identifying different constituents, process parameters, design attributes, and interactions among various subsystems of nanotechnology systems.

\section{The physical significance of various grouping is explained as}

Group 1 contains only one term of five isolated vertices $P_{1}, P_{2} \ldots, P_{N}$ i.e. five subsystems are to be considered together as independent entities. 
Group 2 is absent, as a particular subsystem has no interactions with itself.

Group 3 has ten terms, each term is set of one dyad, $\mathbf{P}_{\mathbf{i j}}$, or two subsystem loop i.e. $\mathbf{P}_{\mathrm{ij}} \mathbf{P}_{\mathrm{ij}}$ and three independent subsystems (dyad is a system of two subsystems $i$ and $j$, considered as one entity) measures of remaining ( $\mathrm{N}-2)$ unconnected elements.

Group 4 has 20 terms in all. Each term has a set of one 3-subsystem loop $P_{i j} P_{j k} P_{k i}$ and $P_{i k} P_{k j} P_{j i}$ independent subsystems measure of the remaining $(\mathrm{N}-3)$ unconnected elements.

Group 5 has two subgroups: Group 5(i) has fifteen terms each term is a subset of two independent dyads or two-subsystem loops and one independent subsystem (i.e. $\mathrm{P}_{\mathrm{ij}} \mathrm{P}_{\mathrm{ji}}$ and $\mathrm{P}_{\mathrm{kl}}$ $\mathrm{P}_{\mathrm{lk}}$ ) Group 5 (ii) has thirty terms each term is a set of 4-subsystem loop $\mathrm{P}_{\mathrm{ij}} \mathrm{P}_{\mathrm{jk}} \mathrm{P}_{\mathrm{kl}} \mathrm{P}_{\mathrm{li}}$ and one independent subsystem $\mathrm{P}_{\mathrm{il}} \mathrm{P}_{\mathrm{lk}} \mathrm{P}_{\mathrm{kj}} \mathrm{P}_{\mathrm{ji}}$.

Group 6 has again two subsystems: Group 6 (i) have one 3-subsystem loop and dyad or twosubsystem loop (i.e. $\mathrm{P}_{\mathrm{kl}} \mathrm{P}_{\mathrm{lm}} \mathrm{P}_{\mathrm{mk}}$ ) or its pair (i.e. $\mathrm{P}_{\mathrm{km}} \mathrm{P}_{\mathrm{ml}} \mathrm{P}_{\mathrm{lk}}$ ). Group 6 (ii) has twenty-four 5subsystem loops (i.e. $\mathrm{P}_{\mathrm{ij}} \mathrm{P}_{\mathrm{jk}} \mathrm{P}_{\mathrm{kl}} \mathrm{P}_{\mathrm{lm}} \mathrm{P}_{\mathrm{mi}}$ ) or its pair (i.e. $\mathrm{P}_{\mathrm{im}} \mathrm{P}_{\mathrm{ml}} \mathrm{P}_{\mathrm{lk}} \mathrm{P}_{\mathrm{kj}} \mathrm{P}_{\mathrm{ji}}$ ). In general, 5-subsystem permanent functions have 5! i.e. 120 terms arranged in $(\mathrm{N}+1)$ groups give the graphical interpretation for visual understanding, analysis, and improvement of the nanotechnology system. It is, therefore, possible for designers as well as manufacturers to carry out SWOT (Strength-weakness-opportunities-threats) analysis of their complete nanotechnology system and take strategic decisions to their advantage as per policy.

\subsubsection{Evaluation of $P_{i}$}

The diagonal elements of the matrix in Equation (5), corresponds to five subsystems that constitute the nanotechnology system. The values of these diagonal elements, $\mathbf{P}_{\mathbf{1}}, \mathbf{P}_{\mathbf{2}} \ldots \mathbf{P}_{\mathbf{5}}$ are calculated as:

$\mathrm{P}_{1}=\operatorname{per}\left(\mathrm{E}_{1}\right) ; \mathrm{P}_{2}=\operatorname{per}\left(E \mathrm{P}_{2}\right) ; \mathrm{P}_{3}=\operatorname{per}\left(\mathrm{E}_{3}\right) ; \mathrm{P}_{4}=\operatorname{per}\left(\mathrm{E} \mathrm{P}_{4}\right) ; \mathrm{P}_{5}=\operatorname{per}\left(\mathrm{EP}_{5}\right) ;$

Where, $\mathrm{E}_{1}, \mathrm{E}_{2}, \mathrm{E}_{3}, \mathrm{E}_{4}$, and $\mathrm{EP}_{5}$ are variable permanent matrices for five subsystems of the nanotechnology system.

The procedure for calculating $\mathbf{P}_{1}, \mathbf{P}_{2}, \mathbf{P}_{3} \ldots \ldots \ldots . . . \mathbf{P}_{5}$ is the same as for calculating per (E) of Equation (6a). Digraph representation of five subsystems is drawn first separately to obtain their matrix Equations i.e. $E P_{i}$ S and their permanent functions $\mathbf{P}_{\mathbf{i}} \mathbf{S}, \mathbf{i}=\mathbf{1}, \mathbf{2}, \mathbf{3} \ldots \mathbf{5}$. The off-diagonal terms $\mathbf{P}_{\mathbf{i j}}(\mathbf{i}$, $\mathbf{j = 1 , 2}, \ldots . . ., 5$ ) of matrix Equation (5) gives the connections between the systems $P_{\mathrm{i}}$ and $\mathrm{P}_{\mathrm{j}}$. Depending upon the type of structural analysis, $\mathbf{P}_{\mathbf{i j}}$ represented as a multinomial, graph matrix, or by some analytical model. To get the exact degree of interactions, interconnections, dependencies, connectivity, etc. between subsystems or sub-sub systems, one has to consider the views of technical team experts. The team of experts selected from design, manufacturing, 
chemistry, material science, etc. to consider all issues concurrently involved from engineering, science, technology, and business strategy point of view. The final decision on the values of $\mathbf{P}_{\mathbf{i}} \mathbf{S}$ and $\mathbf{P}_{\mathrm{ijj}} \mathbf{S}$ is taken on the recommendations of the team. Thus, the following top-down approach and the step-by-step procedure is given below gives the complete structural analysis of the nanotechnology system.

\subsection{Nanotechnology system analysis}

The methodology described in the previous sections for a complete analysis of a nanotechnology system also the visualization model of the proposed methodology is shown in Figure 6. The values (or functions) of interactions, $\mathbf{P}_{\mathrm{ij}}(\mathbf{i}, \mathbf{j}=\mathbf{1}, \mathbf{2}, \mathbf{3} \ldots \mathbf{N})$ Between different subsystems, $\mathbf{P}_{1}, \mathbf{P}_{2} \ldots \mathbf{P}_{\mathbf{N}}$ is written as a multinomial or matrix, depending upon the type of interactions/reactions between the two subsystems. The sub-sub systems again treated as systems, where every sub-subsystem is a system in itself. Following the above procedure, these subsystems are broken down into sub-sub systems and different graphs, matrix, and permanent representations are obtained. Depending upon the depth of analysis required, the process could be taken to the constituent level and further. In certain cases, it would be possible to evaluate $\mathbf{P}_{\mathbf{i j}}$ s experimentally or using available mathematical models. With the help of this data, complete multinomial for the nanotechnology system is evaluated. Work is in progress to carry out performance analysis of any nanotechnology system from different perspectives using the structural model presented in the article.

\subsection{Generalization of the model}

The variable permanent function (VPF) being the characteristic of the nanotechnology system for any industrial nanoproducts and is a powerful tool for its analysis. The VPFNANOTECHNOLOGY system expression, which corresponds to five-factor digraph, is written in compact sigma $(\Sigma)$ form:

VPF-NANOTECHNOLOGY $=\operatorname{Per}(\mathbf{E})=\prod_{1}^{5}+\sum_{\mathrm{i}} \sum_{\mathrm{j}} \sum_{\mathrm{k}} \sum_{\mathrm{l}} \sum_{\mathrm{m}} \cdot\left(\mathrm{P}_{\mathrm{ij}} \mathrm{P}_{\mathrm{ji}}\right) \mathrm{P}_{\mathrm{k}} \mathrm{P}_{\mathrm{l}} \mathrm{P}_{\mathrm{m}}+\sum_{\mathrm{i}} \sum_{\mathrm{j}} \sum_{\mathrm{k}} \sum_{\mathrm{l}} \sum_{\mathrm{m}} \cdot\left(\mathrm{P}_{\mathrm{ij}} \mathrm{P}_{\mathrm{jk}}\right.$ $\left.\mathrm{P}_{\mathrm{ki}}+\mathrm{P}_{\mathrm{ik}} \mathrm{P}_{\mathrm{kj}} \mathrm{P}_{\mathrm{ji}}\right) \mathrm{P}_{\mathrm{l}} \mathrm{P}_{\mathrm{m}}+\left(\sum_{\mathrm{i}} \sum_{\mathrm{j}} \sum_{\mathrm{k}} \sum_{\mathrm{l}} \sum_{\mathrm{m}} \cdot\left(\mathrm{P}_{\mathrm{ij}} \mathrm{P}_{\mathrm{jk}}\right)\left(\mathrm{P}_{\mathrm{kl}} \mathrm{P}_{\mathrm{lk}}\right) \mathrm{P}_{\mathrm{m}}+\sum_{\mathrm{i}} \sum_{\mathrm{j}} \sum_{\mathrm{k}} \sum_{\mathrm{l}} \sum_{\mathrm{m}} \cdot\left(\left(\mathrm{P}_{\mathrm{ij}} \mathrm{P}_{\mathrm{jk}} \mathrm{P}_{\mathrm{kl}} \mathrm{P}_{\mathrm{li}}\right)+\left(\mathrm{P}_{\mathrm{il}} \mathrm{P}_{\mathrm{lk}} \mathrm{P}_{\mathrm{kj}}\right.\right.\right.$ $\left.\left.\left.\mathrm{P}_{\mathrm{ji}}\right)\right) \mathrm{P}_{\mathrm{m}}\right)+\sum_{\mathrm{i}} \sum_{\mathrm{j}} \sum_{\mathrm{k}} \sum_{\mathrm{l}} \sum_{\mathrm{m}} \cdot\left(\mathrm{P}_{\mathrm{ij}} \mathrm{P}_{\mathrm{ji}}\left(\mathrm{P}_{\mathrm{kl}} \mathrm{P}_{\mathrm{lm}} \mathrm{P}_{\mathrm{mk}}+\mathrm{P}_{\mathrm{km}} \mathrm{P}_{\mathrm{ml}} \mathrm{P}_{\mathrm{lk}}\right)+\sum_{\mathrm{i}} \sum_{\mathrm{j}} \sum_{\mathrm{k}} \sum_{\mathrm{l}} \sum_{\mathrm{m}} \cdot\left(\mathrm{P}_{\mathrm{ij}} \mathrm{P}_{\mathrm{jk}} \mathrm{P}_{\mathrm{kl}} \mathrm{P}_{\mathrm{lm}} \mathrm{P}_{\mathrm{mi}}+\right.\right.$ (7) $\left.\mathrm{P}_{\mathrm{ml}} \mathrm{P}_{\mathrm{lk}} \mathrm{P}_{\mathrm{kj}} \mathrm{P}_{\mathrm{ji}}\right)$ )

The Equation (7) is a generalized mathematical expression in a symbolic form corresponding to five-factor digraph representation. It ensures the estimates of the nanotechnology system for any industrially fabricated nanoproduct. Equation (7) contains terms arranged in ( $N+1)$ groups, where $\mathrm{N}$ is the number of subsystems, in that case, it is 5 . 


\subsubsection{Generalization of the present methodology}

Suppose, the system consists of $\mathrm{N}$ subsystems and is represented as a digraph, then the most general way of matrix representation is shown below. The matrix is known as a variable permanent matrix (VPM) corresponding to $\mathrm{N}$ subsystems is given by Equation (8).

\begin{tabular}{|c|c|c|c|c|c|}
\hline 1 & 2 & 3 & - & $\mathrm{N}$ & Components \\
\hline$P_{1}$ & $P_{12}$ & $P_{13}$ & - & $P_{1 N}$ & 1 \\
\hline$P_{21}$ & $\mathrm{P}_{22}$ & $\mathrm{P}_{23}$ & - & $\mathrm{P}_{2 \mathrm{~N}}$ & 2 \\
\hline $\mathrm{P}_{31}$ & $\mathrm{P}_{32}$ & $\mathrm{P}_{33}$ & - & $\mathrm{P}_{3 \mathrm{~N}}$ & 3 \\
\hline - & - & - & - & - & - \\
\hline $\mathrm{P}_{\mathrm{N} 1}$ & $\mathrm{P}_{\mathrm{N} 2}$ & $\mathrm{P}_{\mathrm{N} 3}$ & - & $P_{N}$ & $\mathrm{~N}$ \\
\hline
\end{tabular}

Permanent for the above matrix, i.e. Per (E) is called variable permanent function (VPF). The VPF for the above matrix is written in sigma form as Variable permanent function (VPF):

$$
\begin{aligned}
& \operatorname{Per}(\mathrm{E})=\prod_{\mathrm{a}=1}^{\mathrm{N}}+\sum_{\mathrm{i}} \sum_{\mathrm{j}} \sum_{\mathrm{k}} \ldots \ldots \ldots \sum_{\mathrm{N}} \cdot\left(\mathrm{P}_{\mathrm{ij}} \mathrm{P}_{\mathrm{ji}}\right) \mathrm{P}_{\mathrm{k}} \mathrm{P}_{\mathrm{l}} \ldots \ldots \mathrm{P}_{\mathrm{N}}+\sum_{\mathrm{i}} \sum_{\mathrm{j}} \sum_{\mathrm{k}} \ldots \ldots \ldots \sum_{\mathrm{N}} \cdot\left(\mathrm{P}_{\mathrm{ij}} \mathrm{P}_{\mathrm{jk}} \mathrm{P}_{\mathrm{ki}}+\right. \\
& \left.\mathrm{P}_{\mathrm{ik}} \mathrm{P}_{\mathrm{kj}} \mathrm{P}_{\mathrm{ji}}\right) \mathrm{P}_{\mathrm{l}} \mathrm{P}_{\mathrm{m}} \ldots \ldots \ldots \ldots \mathrm{P}_{\mathrm{N}}+\left(\sum_{\mathrm{i}} \sum_{\mathrm{j}} \sum_{\mathrm{k}} \ldots \ldots \ldots \sum_{\mathrm{N}} \cdot\left(\mathrm{P}_{\mathrm{ij}} \mathrm{P}_{\mathrm{jk}}\right)\left(\mathrm{P}_{\mathrm{kl}} \mathrm{P}_{\mathrm{lk}}\right) \mathrm{P}_{\mathrm{m}} \mathrm{Pn} \cdots \cdots \cdots \mathrm{P}_{\mathrm{N}}+\sum_{\mathrm{i}} \sum_{\mathrm{j}} \sum_{\mathrm{k}} \ldots \ldots \ldots \sum_{\mathrm{N}} \cdot\left(\mathrm{P}_{\mathrm{ij}}\right.\right. \\
& \left.\left.\left.\mathrm{P}_{\mathrm{jk}} \mathrm{P}_{\mathrm{kl}} \mathrm{P}_{\mathrm{li}}\right)+\left(\mathrm{P}_{\mathrm{il}} \mathrm{P}_{\mathrm{lk}} \mathrm{P}_{\mathrm{kj}} \mathrm{P}_{\mathrm{ji}}\right)\right) \mathrm{P}_{\mathrm{m}} \mathrm{Pn} \ldots \ldots \ldots \mathrm{P}_{\mathrm{N}}\right)+\left(\sum_{\mathrm{i}} \sum_{\mathrm{j}} \sum_{\mathrm{k}} \ldots \ldots \ldots \sum_{\mathrm{N}} \cdot\left(\mathrm{P}_{\mathrm{ij}} \mathrm{P}_{\mathrm{ji}}\right)\left(\mathrm{P}_{\mathrm{kl}} \mathrm{P}_{\mathrm{lm}} \mathrm{P}_{\mathrm{mk}}+\mathrm{P}_{\mathrm{km}} \mathrm{P}_{\mathrm{ml}} \mathrm{P}_{\mathrm{lk}}\right) \mathrm{Pn}\right. \\
& \left.\mathrm{Pa} \cdots \cdots \cdots \cdot \mathrm{P}_{\mathrm{N}}+\sum_{\mathrm{i}} \sum_{\mathrm{j}} \sum_{\mathrm{k}} \ldots \ldots \ldots \sum_{\mathrm{N}} \cdot\left(\mathrm{P}_{\mathrm{ij}} \mathrm{P}_{\mathrm{jk}} \mathrm{P}_{\mathrm{kl}} \mathrm{P}_{\mathrm{lm}} \mathrm{P}_{\mathrm{mi}}+\mathrm{P}_{\mathrm{im}} \mathrm{P}_{\mathrm{ml}} \mathrm{P}_{\mathrm{lk}} \mathrm{P}_{\mathrm{kj}} \mathrm{P}_{\mathrm{ji}}\right) \mathrm{Pn} \mathrm{Po} \cdots \cdots \cdots \cdot \mathrm{P}_{\mathrm{N}}\right)+\cdots \cdots \cdots \cdots
\end{aligned}
$$

The number and composition of groups and subgroups are the same as discussed earlier. So it is possible to write the permanent function of any nanotechnology system. It is noted that permanent functions contain $\mathrm{N}$ ! terms only provided $\mathrm{P}_{\mathrm{ij}} \mathrm{S}$ are not 0 . In certain cases, the designer and/or manufacturer team decide that some of $\mathrm{P}_{\mathrm{ij}} \mathrm{S}$ are 0 . The substitution of corresponding $\mathrm{P}_{\mathrm{ij}} \mathrm{S}$ equal to 0 in general permanent function (Equation (9)) or general VPM (Equation (8)) gives the exact number of terms with modified permanent function.

\subsection{Comparison and Validation of Proposed Methodology}

The nanotechnology system is represented as a system that consists of five subsystems, which affect the properties and performance of the final system. The five-subsystem nanotechnology system is modeled as a multinomial, permanent function. Different nanotechnology systems based on different areas have a different number of terms in different groups and subgroups of their permanent functions. The similarity or dissimilarity between any nanotechnology systems is obtained by comparing their permanents. Using the proposed methodology, identification of the nanotechnology system and its comparison with other nanotechnology system is based on the analysis carried out with the help of VPF-NANOTECHNOLOGY. Two nanotechnology systems are similar from subsystems and their interactions viewpoint if their digraphs are 
isomorphic. Two nanotechnology systems digraph is isomorphic if having identical VPFNANOTECHNOLOGY. It means that not only the number of terms in each grouping/subgrouping is the same, but the values are the same. Based on that, nanotechnology system identification set for any system is written by Equation (10) as:

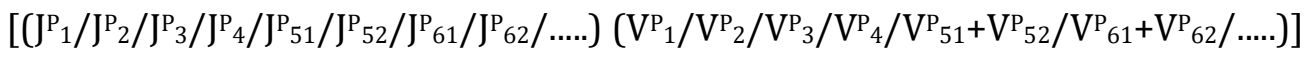

Where $J_{\mathrm{i}} \mathrm{P}_{\mathrm{i}}$ represents the total number of terms in an ith grouping. $\mathrm{J}_{\mathrm{ij}}$ represents the total number of terms in a jh grouping. In case, there is no subgrouping, $\mathrm{J}_{\mathrm{ij}}$ is the same as $\mathrm{JP}_{\mathrm{i}}$. Similarly, $\mathrm{V}_{\mathrm{i}}$ is the value of $\mathrm{i}^{\text {th }}$ grouping. $\mathrm{V}_{\mathrm{ij}}$ is the numerical values of the $\mathrm{P}_{\mathrm{i}} \mathrm{S}$ and $\mathrm{P}_{\mathrm{ij}} \mathrm{S}$ that are substituted in the subgrouping or grouping to obtain $\mathrm{VP}_{\mathrm{ij}}$. The sub-groupings are arranged in decreasing order of size (i.e. number of elements in a loop). In general, two nanotechnology systems would not be isomorphic from the viewpoint of interactions among subsystems. A comparison is carried out based on the coefficient of similarity. The coefficient is derived from the structure, i.e. VPFNANOTECHNOLOGY, and compares two nanotechnology systems or a set of nanotechnology systems based on similarity or dissimilarity. If the values of distinct terms in the $\mathrm{j}^{\text {th }}$ subgrouping of an $i^{\text {th }}$ grouping of VPF-NANOTECHNOLOGY of two nanotechnology systems under consideration are denoted by $\mathrm{VP}_{\mathrm{ij}}$ and $\mathrm{V}^{\prime} \mathrm{P}_{\mathrm{ij}}$ then two criteria are proposed as follows:

Criterion 1: The coefficient of dissimilarity $\mathrm{CP}_{\mathrm{d}-1}$ based on Criterion 1 is proposed as

$\mathrm{C}_{\mathrm{d}-1}=1 / \mathrm{Y}_{1} \sum_{\mathrm{i}} \sum_{\mathrm{j}} \varnothing \mathrm{ij}, \quad$ Where, $\mathrm{Y}_{1}=\max \left(\sum_{\mathrm{i}} \sum_{\mathrm{j}}\left[\mathrm{V}_{\mathrm{ij}}\right]\right.$ and $\left.\sum_{\mathrm{i}} \sum_{\mathrm{j}}\left[\mathrm{V}^{\prime} \mathrm{P}_{\mathrm{ij}}\right]\right)$

When, sub-groupings are absent $V_{i j}=V^{P_{i}}$ and $V^{\prime} P_{i j}=V^{\prime} P_{i}, \phi_{i j}=\left[V^{P_{i j}}-V^{\prime} P_{i}\right]$ when sub-grouping exists and $\phi_{\mathrm{ij}}=\left[\mathrm{V}_{\mathrm{i}}-\mathrm{V}^{\prime} \mathrm{P}_{\mathrm{i}}\right]$ when sub-groupings are absent.

Criterion 2: The coefficient of dissimilarity $\mathrm{CP}_{\mathrm{d}-2}$ based on Criterion 2 is proposed as

$\left(\mathrm{C}_{\mathrm{d}-2}=1 / \mathrm{Y}_{1} \sum_{\mathrm{i}} \sum_{\mathrm{j}} \cdot \phi^{2 \mathrm{ij}}\right)^{1 / 2}$

Where, $\phi_{\mathrm{ij}}$ is the same as described above and

$\mathrm{Y}_{2}=\max \left(\sum_{\mathrm{i}} \sum_{\mathrm{j}} \cdot\left(\mathrm{V}_{\mathrm{ij}}\right)^{2}\right.$ and $\left.\sum_{\mathrm{i}} \sum_{\mathrm{j}} \cdot\left(\mathrm{V}^{\prime} \mathrm{P}_{\mathrm{ij}}\right)^{2}\right)$ 
When, sub-groupings are absent $V_{i j}=V^{P_{i}}$ and $\left(V^{\prime} P_{i j}\right)=V^{\prime} P_{i}$, using Equations (11) and (12), the coefficient of similarity is given as $\mathbf{C P}_{\mathrm{s}-\mathbf{1}}=\mathbf{1}-\mathbf{C}_{\mathrm{d}-\mathbf{1}} ; \mathbf{C}_{\mathrm{s}-2}=\mathbf{1}-\mathbf{C}_{\mathrm{d}-\mathbf{2}}$

Where $\mathbf{C}_{\text {s-1 }}$ and $\mathbf{C}_{\text {s-2 }}$ are the coefficients of similarity between two nanotechnology systems under consideration based on criterion 1 and 2. It is noted that the coefficient of similarity and dissimilarity lies in the range between 0 and 1, if two nanotechnology systems are isomorphic or completely similar, then their coefficient of similarity is 1 and the coefficient of dissimilarity is 0 . Similarly, if two nanotechnology systems are completely dissimilar, then their coefficient of similarity is 0 and the coefficient of dissimilarity is 1 . In this way, any system is to be validated based upon the selection of the number of terms in a group and also upon the coefficient of similarity and dissimilarity between the two systems. This work is extended to do the Sensitivity analysis of attribute(s) - one at a time and in combination is an important issue for $\mathrm{R}$ $\&$ D of new nanomaterials for different applications of nanotechnology systems.

\subsection{The usefulness of the proposed methodology}

The methodology is dynamic as constituents and bonding are changed without any difficulty. The approach helps to express the nanotechnology system in quantitative terms, which has more often been expressed in qualitative terms. The procedure helps to compare different nanotechnology systems in terms of its characteristics and rate them for a particular application. It is hoped that the methodology provides a new direction in the research attempts towards global projects of quantitative structure-activity relationship (QSAR) and quantitative structure properties relationship (QSPR). The present work is an attempt towards the development of a complete methodology for virtual fabrication and design of nanoproducts. It helps to model the complete nanotechnology system which helps the different researchers, manufacturers, and designers to create different nanoproducts in a minimum possible time.

\section{Conclusions}

In the paper, an attempt is made to develop an integrated system model for the structure of the nanotechnology system by considering all the interactions between the constituents and processes using the digraph and matrix approach. It shows how these interactions affect the process parameters, design, and production attributes of nanotechnology products. The system methodology consists of a nanotechnology system digraph, nanotechnology system matrix, and nanotechnology permanent function. The nanotechnology system's permanent function helps in characterizing the structure of the nanotechnology system and to determine the nanotechnology system index. The multi-attribute (structural constituents) characterization of the nanotechnology system into a single numerical index is done. The numerical index of the 
nanotechnology system derived from a multinomial is used for comparison, rating, and optimum selection. The present work emphasizes the numerical methodology of the nanotechnology system which optimizes the design and the production parameters. The generalized methodology is proposed to a model system consisting of $\mathrm{N}$ subsystems and their interactions. The study gives criterion on how to compare two nanotechnology systems with the help of permanent function on a structured basis. The derivation and importance of numerical index of nanotechnology system are presented in future work with illustrative examples also an attempt is made in future publications to correlate nanotechnology performance characteristics with nanotechnology structural models reported in the article.

\section{References}

1. Mao, L. F. (2011). Study of the conduction band offset alignment caused by Oxygen vacancies in sio2 layer and its effects on the gate leakage current in nano-MOSFETs, Iranian Journal of Science and Technology Transaction of Electrical Engineering, 35 E1:1-11.

2. Ghaffarpour, J.S. Ahmadi, N.A. Mortazavi, S.M., and Vossough, S. (2011). Rutting and fatigue behavior of nano clay modified bitumen, Iranian Journal of Science and Technology, Transaction of civil Engineering, 35(C2):277-281.

3. Sadrmomtazi, A., and Fasihi, A. (2010). Influence of polypropylene fibers on the performance of nano-SiO2- incorporated mortar, Iranian Journal of Science and Technology Transaction B: Engineering, 34 B4:385-395.

4. Lubick, N. (2008). Silver socks have a cloudy lining. Environ, Sci. Technol, 42(11):3910.

5. Cavalcanti, A. et al. (2007). Medical Nanorobot Architecture Based on Nanobioelectronics, Recent Patents on Nanotechnology, 1(1):1-10.

6. Boukallel, M. et al. (2007). Smart microrobots for mechanical cell characterization and cell convoying, IEEE Trans Biomed Eng, 54(8): 1536-40.

7. Zesch, W. (1997). Multi-degree-of-freedom micro-positioning using stepping principles. Technical report, Swiss Federal Institute of Technology, ETHZ Z. Zurich, Switzerland.

8. Darby, AP., and Pellegrino, S. (1999). Modeling and control of a flexible structure incorporating inertial stick-slip actuators, J Guidance Control Dyn, 22: 36-43.

9. Shanian, A., and Savadogo, O. (2006). TOPSIS multiple-criteria decision support analysis for material selection of metallic bipolar plates for polymer electrolyte fuel cell, J Power Sources, 159:1095-104.

10. Schaffer, E et al. (2000). Electrical induced structure formation and pattern transfer, Nature, 403:874-7.

11. Rao, RV. (2008). A decision-making methodology for material selection using an improved compromise ranking method, Mater Des, 29:1949-54. 
12. Bhaduri, D et al. (2005). Nanolab a tool for evaluating the reliability of defect-tolerant nanoarchitectures, IEEE Transactions on Nanotechnology, 4(4).

13. Offodlle, O.F. et al. (1987). Development of a computer-aided robot selection procedure (CARSP), International Journal of Production Research, 25 (8):1109-1121.

14. Buehler, M.J. (2006). Mesoscale modeling of mechanics of carbon nanotubes: Self-assembly, self-folding, and fracture, J. Mater. Res, 21: 2855-2869.

15. Barnard, A.S. (2010). Modeling of nanoparticles: approaches to morphology and evolution, Reports on Progress in Physics, 73:8.

16. Carpenter, D.T. (1998). A methodology for automated quantitative microstructural analysis of transmission electron micrographs, J. Appl. Phys, 84:5843.

17. Amrute, N.M et al. (2010). Targeted Optimization of a Protein Nanomachine for Operation in Biohybrid Devices, Angewandte Chemie, 122(2): 322-326.

18. Patel, G.M et al. (2010). Nanorobot: A versatile tool in nanomedicine, Journal of Drug Targeting, 14(2):63-67.

19. Wang, J. (2011). Micromachine enables Capture and Isolation of Cancer Cells in Complex Media, Angew Chem In Ed, 50:4161-4165.

20. Fan, H. et al. (2000). Rapid prototyping of patterned functional structures, Nature, 405:5660.

21. Sacks, I. J. et al. (1993). Systems Interaction Results from the Digraph Matrix Analysis of Watts bar Nuclear Power Plant High-Pressure Safety Injection System, Lawrence Livermore National Laboratory, Liver more, USA. Report No. UCRI- 53467, Vol 1, 2, 16-33.

22. Seghal, R. Gandhi, O. P. Angra, S. (2000). Reliability Evaluation and Selection of Rolling Element Bearings, Reliability Engineering and System Safety, 68:39-52.

23. Venkataswamy, R., and Agrawal, V.P. (1995). System and structural analysis of an automobile vehicle: a graph-theoretic approach, International Journal of vehicle design, 16(4/5): 477-503.

24. Mohan, M. Gandhi, O.P., and Agrawal, V.P. (2003). Systems modeling of a coal-based steam power plant, In. Proc. Instn. Mech. Engrs, Part A; Journal of Power and Energy, 217(3):259277.

25. Gandhi, O.P., and Agrawal, V.P. (1992). FEMA: A Digraph and Matrix Approach, Reliability Engineering and System Safety, 35(2):147-158.

26. Sandeep, G. Agrawal, V.P., and Khan, I.A. (2004). A Digraph Approach to TQM Evaluation of an industry, International Journal of Production Research, 42(19):4031-4053.

27. Davis, R.C. (2003). Chemo mechanical surface patterning and functionalization of silicon surfaces using an atomic force microscope, Appl. Phys. Letter Related article, 82(5):808810. 
28. Deo, N. (2000). Graph theory with application to engineering and computer science, Prentice-hall, New Delhi, India.

29. Robinson, D.F., and Foulds, L.R. (1980). Digraphs: Theory and techniques, Gordon and Breach science publisher, London.

30. Jurkat, W.B., and Ryser, H.J. (1966). Matrix factorization of determinants and permanents, Journal of Algebra, 3(1):1-27. 


\section{Figure captions}

Figure 1.Structural constituents of nanotechnology system, Note:-STM:- Scanning tunneling microscopy, DPN:- Dip Pan Nanolithography, AFM:- Atomic Force Microscopy, CVD:- Chemical vapor Deposition, PVD:- Pulsed vapor deposition, EBL:- Electron-beam Lithography, TEM:Transmission electron microscope

Figure 2.Schematic representation of the structure of nanotechnology system

Figure 3.Nanotechnology system digraph.

Figure 4.Undirected graph representation of the structure of nanotechnology system

Figure 5. Graphical representation of permanent function (Equation (6)) for nanotechnology system corresponds to the undirected graph (see Figure 4)

Figure 6.Visualization Model of Proposed Methodology 


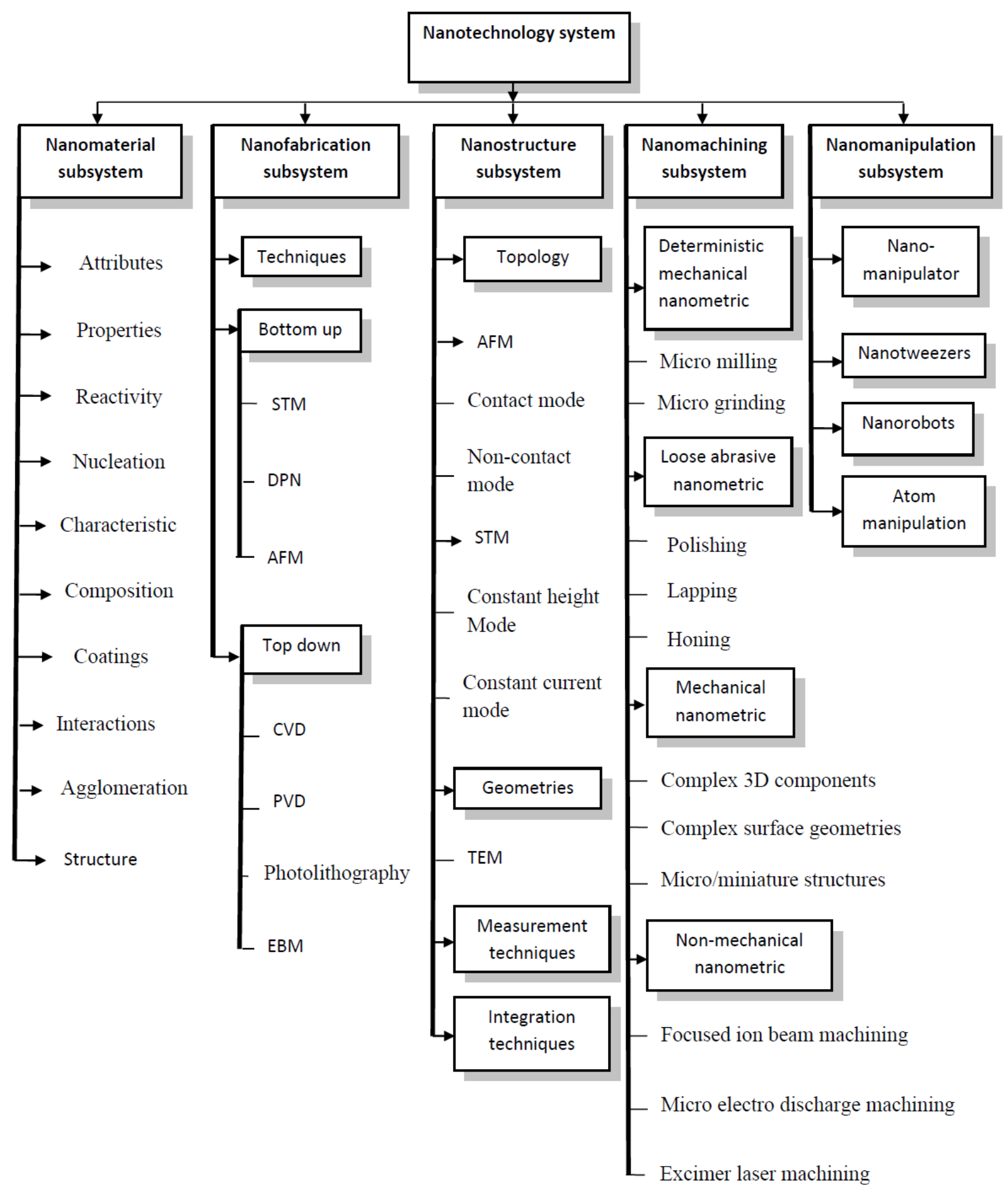

Figure 1 


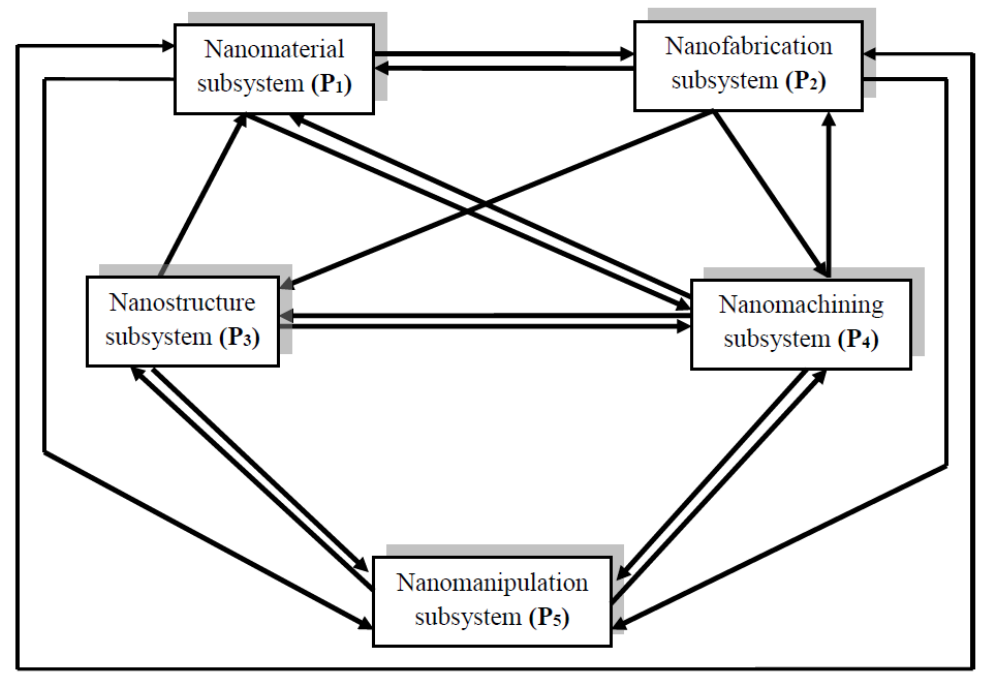

Figure 2

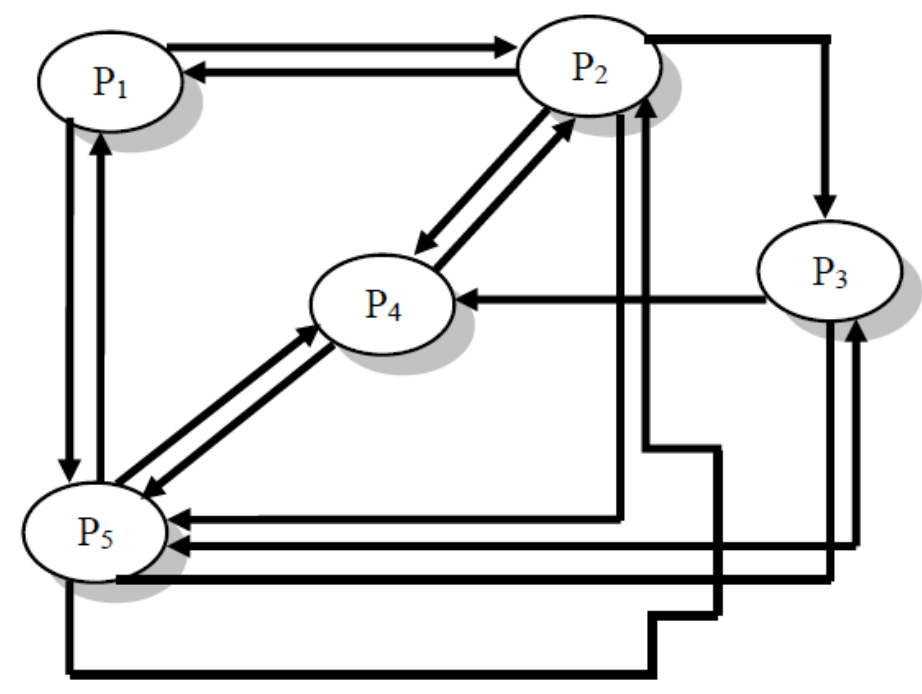

Figure 3

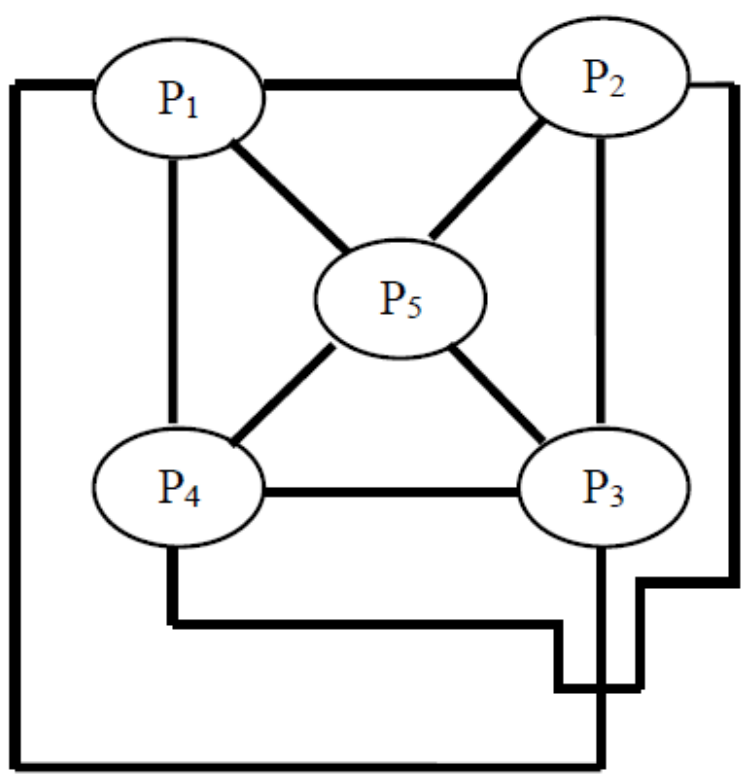

Figure 4 


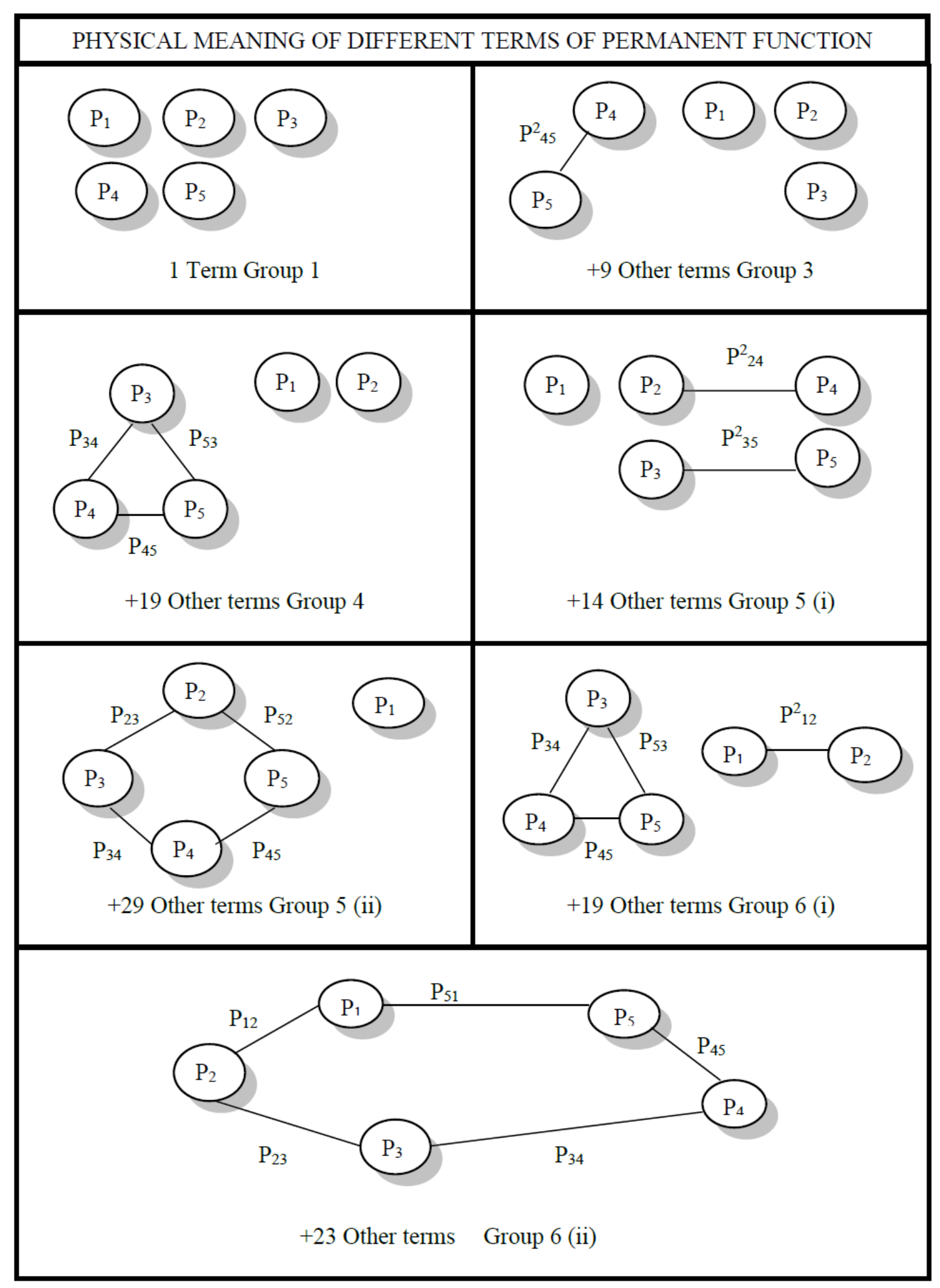

Figure 5 


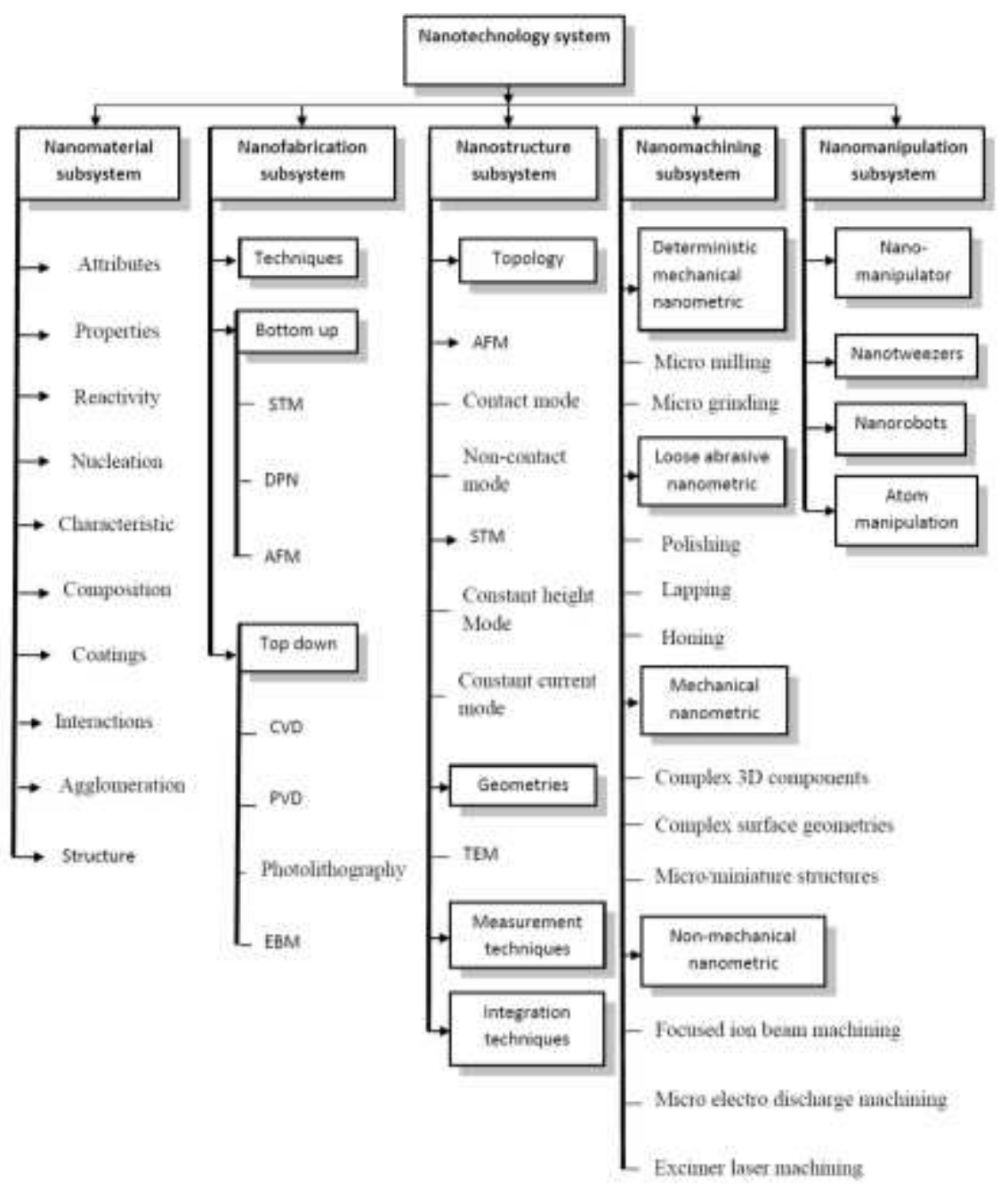

Figure 1

Structural constituents of nanotechnology system, Note:-STM:- Scanning tunneling microscopy, DPN:Dip Pan Nanolithography, AFM:- Atomic Force Microscopy, CVD:- Chemical vapor Deposition, PVD:Pulsed vapor deposition, EBL:- Electron-beam Lithography, TEM:- Transmission electron microscope 


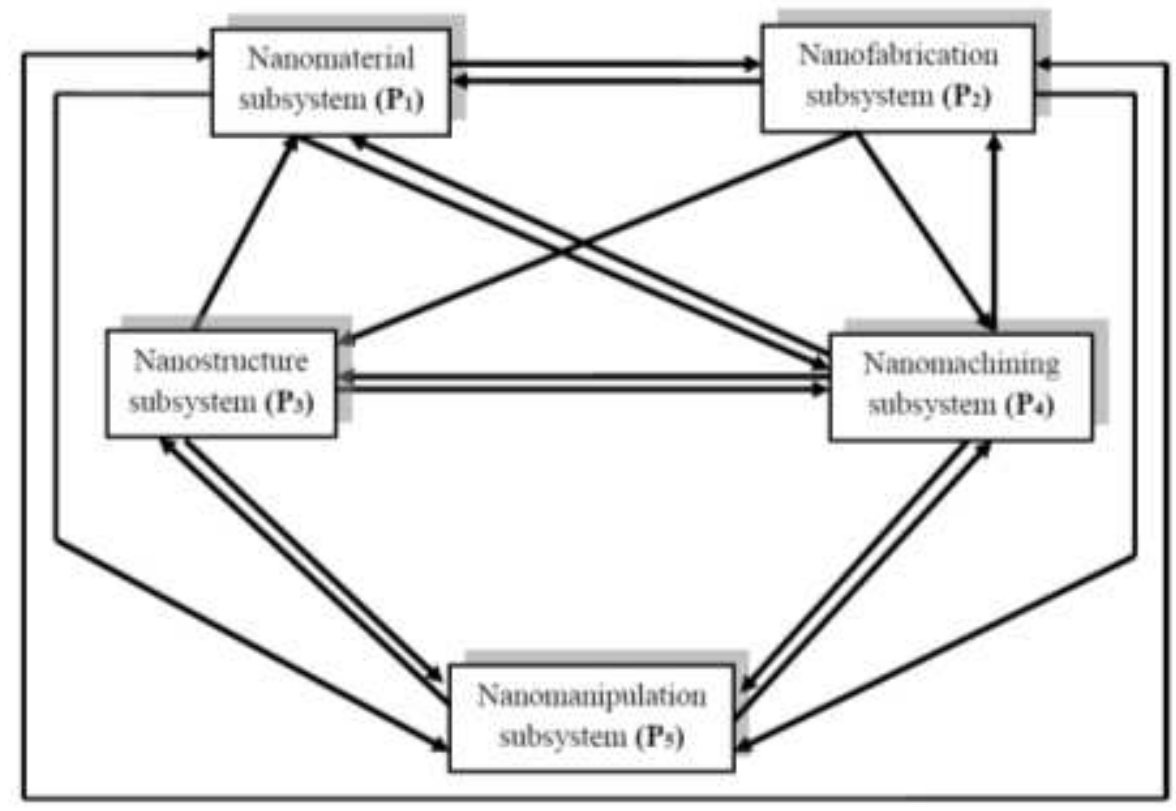

Figure 2

Schematic representation of the structure of nanotechnology system

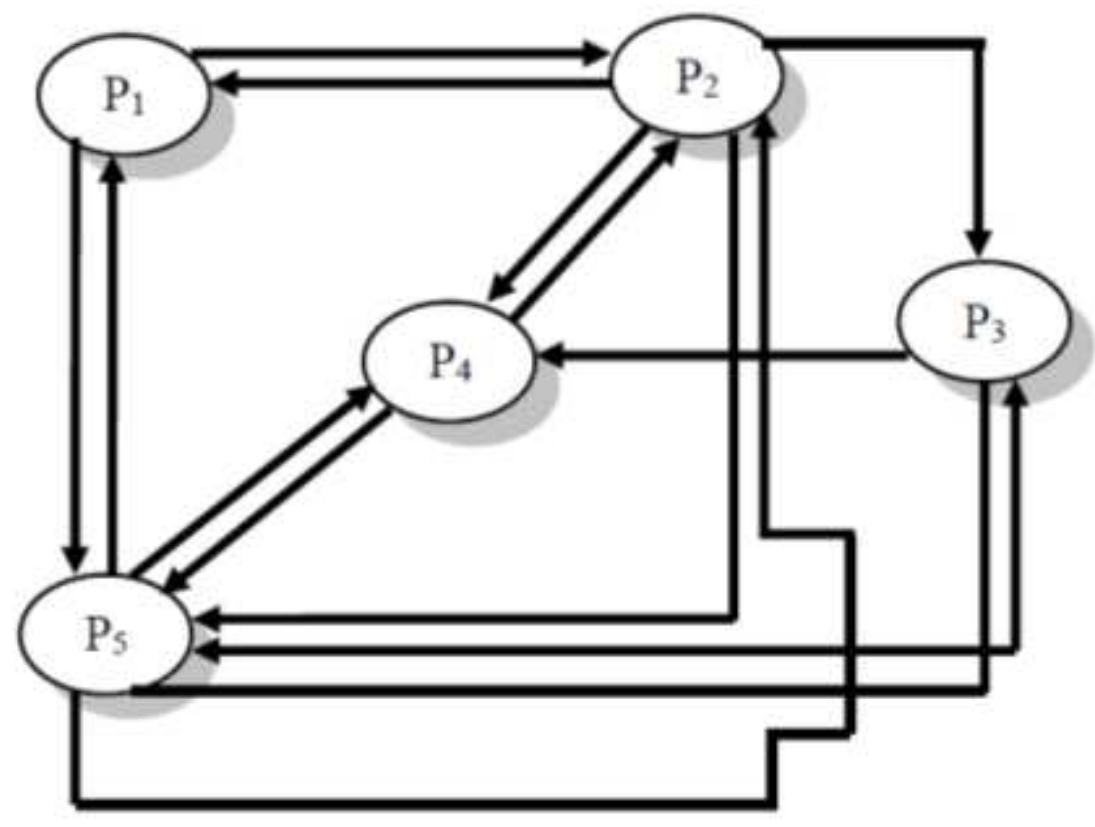

Figure 3

Nanotechnology system digraph. 


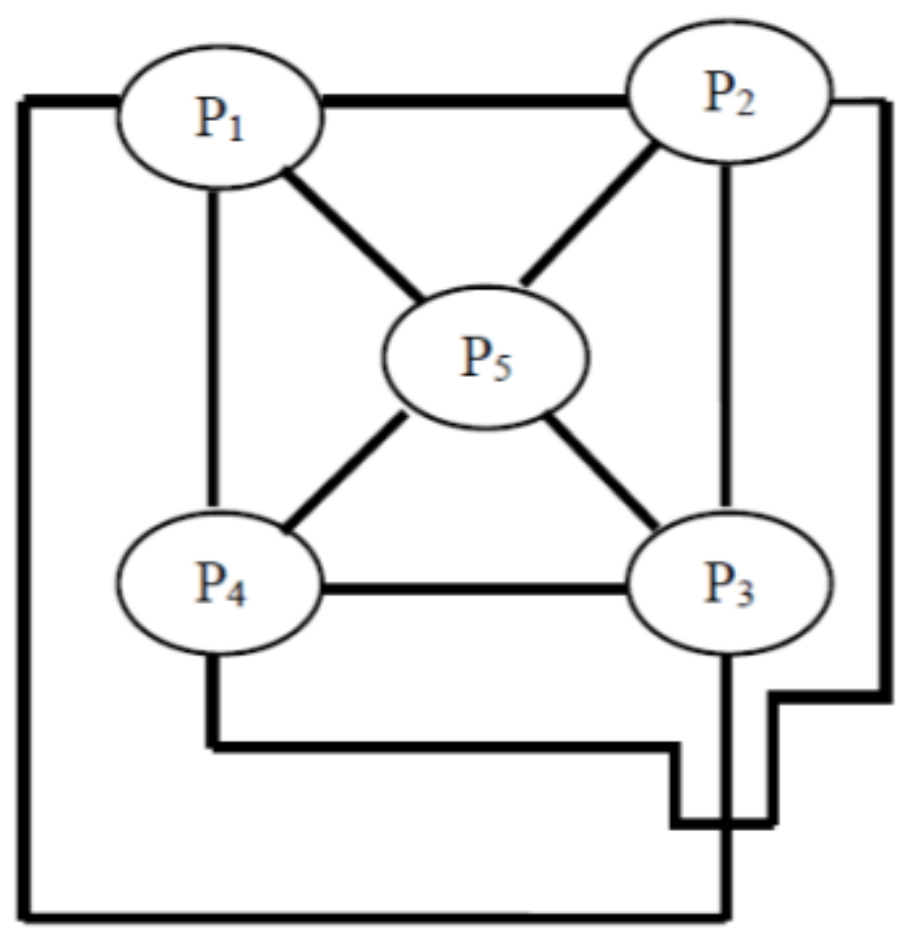

Figure 4

Undirected graph representation of the structure of nanotechnology system 


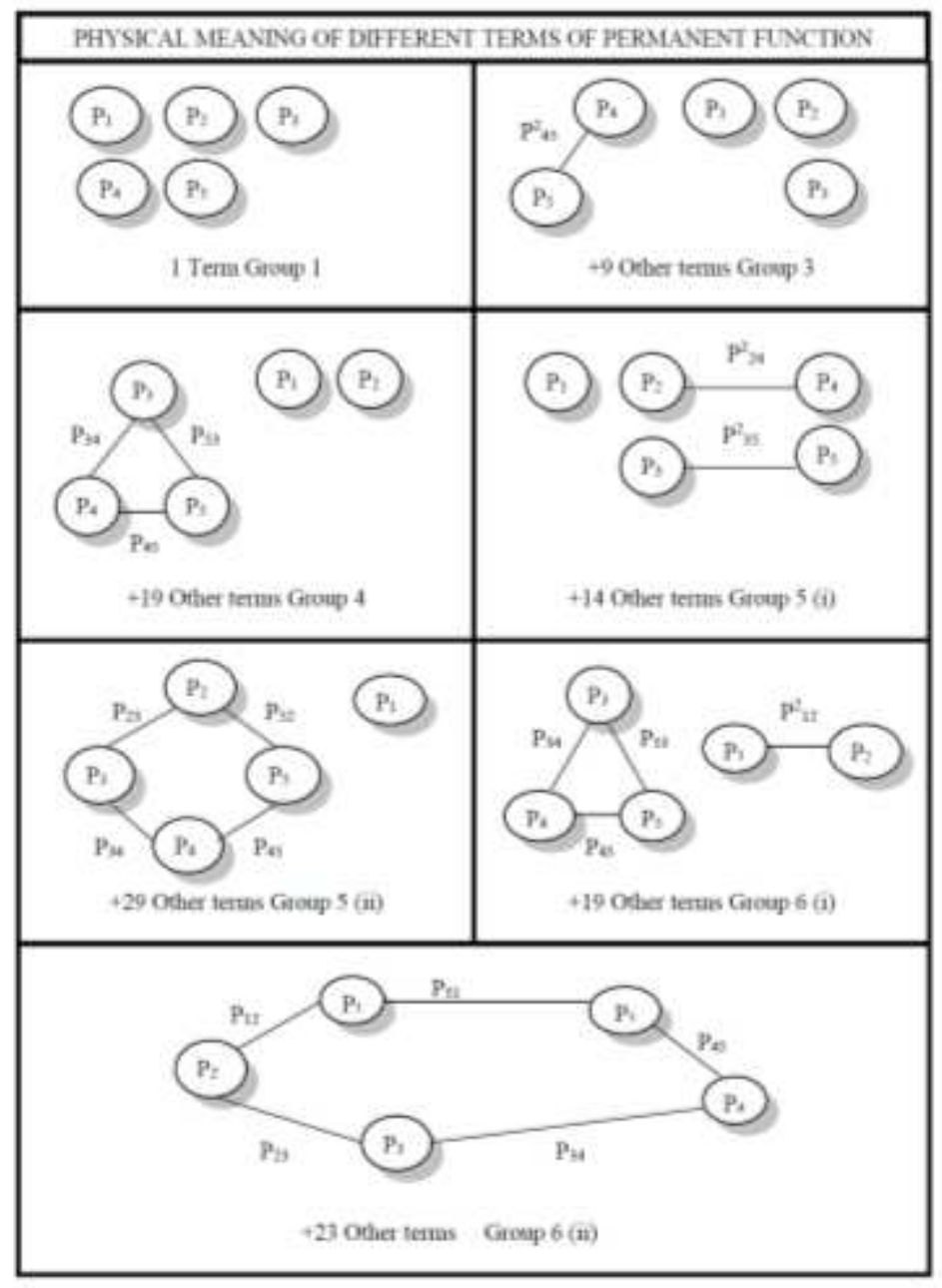

Figure 5

Graphical representation of permanent function (Equation (6)) for nanotechnology system corresponds to the undirected graph (see Figure 4) 


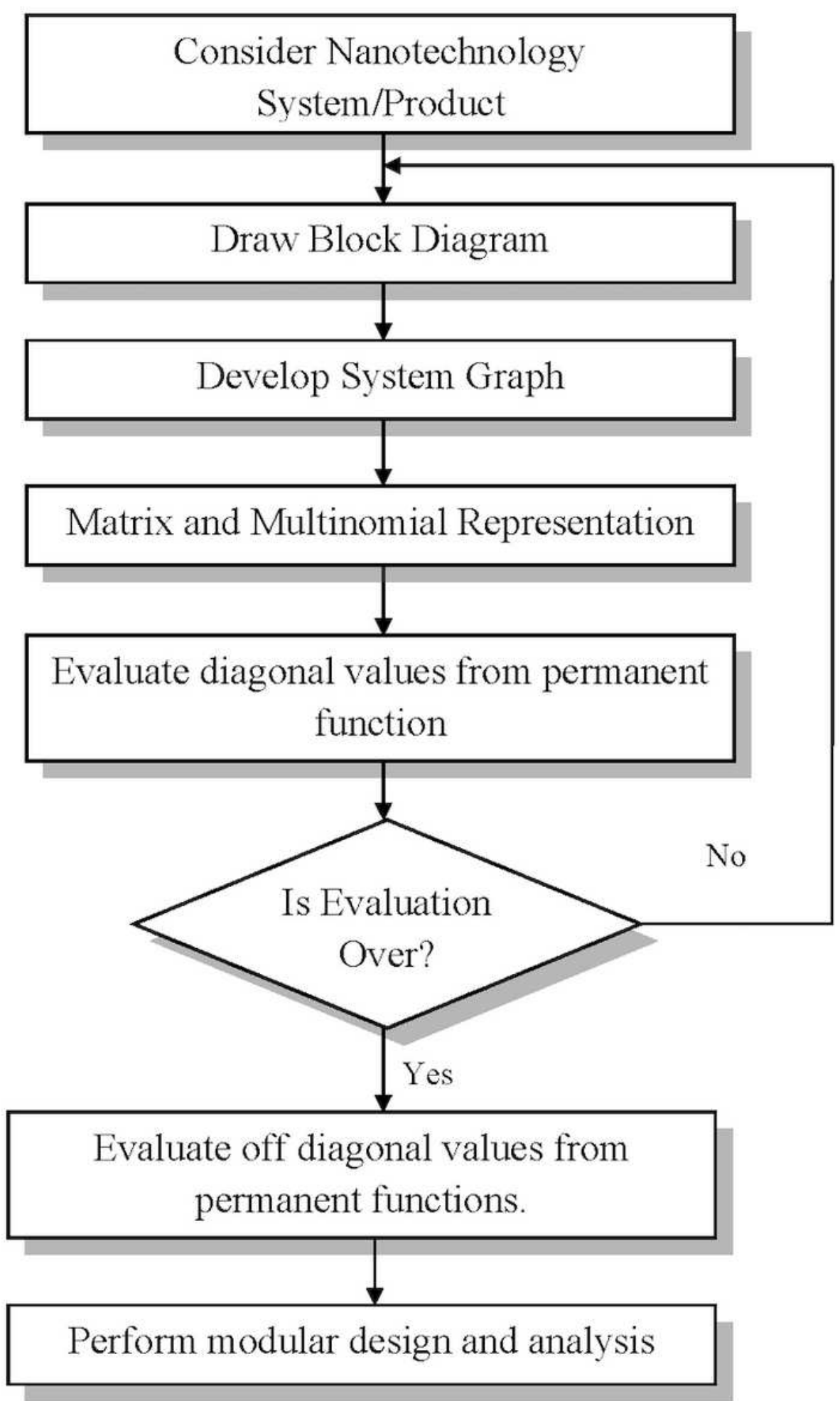

Figure 6

Visualization Model of Proposed Methodology 